Check for updates

Cite this: RSC Adv., 2018, 8, 24633

\title{
Effects of pore size on in vitro and in vivo anticancer efficacies of mesoporous silica nanoparticles
}

\begin{abstract}
Jie Li, † Suqin Shen, $\uparrow$ Fei Kong, Ting Jiang, Cui Tang (D) and Chunhua Yin
Mesoporous silica nanoparticles (MSN) have been widely applied for drug delivery systems. To investigate the effects of pore size on anticancer efficacies, MSN with different pore sizes but similar particle sizes and surface charges were synthesized via a microemulsion method. The pore structures of MSN were characterized by transmission electron microscopy (TEM), small-angle X-ray scattering (SAXS), and $\mathrm{N}_{2}$ adsorption-desorption isotherms. Doxorubicin loaded MSN (DOX/MSN) were prepared and the minimum drug loading capacity was detected in DOX/MSN with a pore size of $2.3 \mathrm{~nm}$ (DOX/MSN2). DOX/MSN with a pore size of $8.2 \mathrm{~nm}$ (DOX/MSN8) showed a comparable drug loading amount in comparison with ones with a pore size of $5.4 \mathrm{~nm}$ (DOX/MSN5). In vitro drug release profiles showed that DOX/MSN5 could release DOX quickly and completely. Compared with DOX/MSN2 and DOX/MSN8, DOX/MSN5 showed the higher cellular uptake and nucleic concentration of DOX in QGY-7703 cells, which led to efficient cell-apoptosis induction and anti-proliferation effect, and thus the optimal in vivo anticancer activities. Taken together, these results highlighted the importance of pore size in anticancer efficacies, which would serve as a guideline in the rational design of MSN for cancer therapy.
\end{abstract}

Received 7th May 2018

Accepted 25th June 2018

DOI: $10.1039 / \mathrm{c} 8 \mathrm{ra03914c}$

rsc.li/rsc-advances drug delivery of various guest molecules. ${ }^{6-8}$ Particularly, the control of pore sizes of MSN is quite essential on account of the confinement effects of pores on both the accessibility of incoming drugs and the release of already-loading drugs. As for drug loading, the pore size should usually be larger than the drug molecules to host the target drug inside the pore space. For example, the pore size of MSN has played an important role in determining the loading capacity of genes. ${ }^{9}$ Moreover, the pore size of MSN has exerted a significant influence on the in vitro release and the cellular uptake of loaded drug. ${ }^{10}$ Although the drug loading of MSN with different pore sizes (5.4-11 nm) enhances with the increase of pore size, MSN with pore size of $8.9 \mathrm{~nm}$ could mediate higher cellular uptake level. ${ }^{11} \mathrm{Jia}$ et al. ${ }^{12}$ have studied the paclitaxel delivery systems based on MSN with various pore sizes $(3-10 \mathrm{~nm})$. They have found that the larger the pore size, the higher drug loading capacity, the faster release rate, and the higher in vitro anticancer activity. To the best of our knowledge, there are few reports about the influences of pore size on the in vivo anticancer efficacies.

In the current study, we aimed to investigate the effects of pore size of MSN on in vitro and in vivo anticancer efficacies of doxorubicin (DOX) loaded into them. A series of DOX loaded MSN (DOX/MSN) with well-defined pore sizes (2.3, 5.4, and 8.2 $\mathrm{nm})$ were designed and developed. Their particle sizes, surface charges, and morphologies were determined. The mesoporous structures were characterized by small-angle X-ray scattering (SAXS) and Barrett, Joyner, and Halenda (BJH) method. The loading capacities and release profiles of DOX were monitored. Besides, cellular uptake, nucleic accumulation, cell-apoptosis
School of Life Sciences, Fudan University, Shanghai 200438, China. E-mail: tangcui@ fudan.edu.cn

$\dagger \mathrm{Jie}$ Li and Suqin Shen contributed equally to this work. 
efficiencies, and anti-proliferation effects of DOX/MSN were conducted in human hepato-carcinoma QGY-7703 cells. In vivo anticancer efficacies of DOX/MSN were studied in tumorbearing mice following intravenous injections.

\section{Materials and methods}

\subsection{Materials, cell culture, and animals}

Tetraethyl orthosilicate (TEOS), cetyltrimethylammonium chloride (CTAC), triethanolamine (TEA), and Hoechst 33258 were purchased from Aladdin Chemistry Co., Ltd. (Shanghai, China). 3-(Trihydroxysilyl) propyl methylphosphonate was purchased from Meryer Chemical Reagent Co., Ltd. (Shanghai, China). Methylthiazolyldiphenyl-tetrazolium bromide (MTT) was obtained from Sigma-Aldrich (St. Louis, MO, USA). DOX was obtained from Zhejiang Hisun Pharmaceutical Co., Ltd. (Zhejiang, China). An Annexin V-FITC/PI apoptosis detection kit was obtained from Yeasen Institute of Biotechnology (Shanghai, China). All other reagents were of analytical reagent grade and purchased from Sinopharm Chemical Reagent Co., Ltd. (Shanghai, China).

Human hepato-carcinoma (QGY-7703) cells were from Chinese Academy of Sciences (Shanghai, China) and cultured in Dulbecco's modified Eagle's medium (DMEM, Gibco, Grand Island, NY, USA) supplemented with $10 \%$ fetal bovine serum (FBS).

Female Kunming mice $(20 \pm 2 \mathrm{~g})$ were provided by the Department of laboratory animal science in Shanghai medical college of Fudan University. All animal procedures were performed in accordance with the Guidelines for Care and Use of Laboratory Animals of Fudan University and experiments were approved by the Animal Ethics Committee of Fudan University.

\subsection{Preparation and characterization of MSN}

The MSN with center-radial pore channels were prepared via a microemulsion method using TEA as a catalyst, CTAC as a template, TEOS as a silica source, and hydrophobic organic solvent (1-octadecene or cyclohexane) as an emulsion agent. A typical synthesis process of the MSN was as follows. ${ }^{13}$ At first, $3.33 \mathrm{~g}$ of CTAC were dispersed in $30 \mathrm{~mL}$ of water and stirred gently at $60{ }^{\circ} \mathrm{C}$ for $2 \mathrm{~h}$ in a round bottom flask in an oil bath and $0.09 \mathrm{~g}$ of TEA were mixed and stirred at $60^{\circ} \mathrm{C}$ for $1 \mathrm{~h}$, then $10 \mathrm{~mL}$ of $20 \%(\mathrm{v} / \mathrm{v})$ TEOS in 1-octadecene was gently added to the upper layer of CTAC-TEA water solution and carefully stirred at $60{ }^{\circ} \mathrm{C}$ for $12 \mathrm{~h}$. Then, the upper solution of 1-octadecene was completely removed and $20 \mu \mathrm{L}$ of 3 -(trihydroxysilyl) propyl methylphosphonate was added to keep the same reaction condition for another $5 \mathrm{~h}$, which was used for phosphate surface modification. ${ }^{\mathbf{1 4}}$ The resultant products were centrifugated and washed with ethanol for several times to remove the residual reactants. Then, the collected products were extracted with $30 \mathrm{~mL}$ of ethanol solution containing $400 \mu \mathrm{L}$ of $\mathrm{HCl}$ at $60{ }^{\circ} \mathrm{C}$ for $12 \mathrm{~h}$ to remove the template. The extracted MSN products were collected by centrifugation and washed with ethanol. The final powder were obtained in a vacuum oven at $30{ }^{\circ} \mathrm{C}$ and stored in the room temperature for the subsequent experiments. The upper oil layer of $10 \mathrm{~mL}$ of $20 \%$ (v/v) TEOS in 1-octadecene was replaced with $10 \mathrm{~mL}$ of $20 \%(\mathrm{v} / \mathrm{v})$ TEOS or $40 \mathrm{~mL}$ of $5 \%(\mathrm{v} / \mathrm{v})$ TEOS in cyclohexane for preparing MSN with different pore sizes but identical particle sizes and surface charges.

Particle sizes and surface charges were detected by dynamic light scattering (DLS) using a Zetasizer Nano (Malvern, UK). The morphology of MSN was performed with a JEM 2011 transformation electron microscopy (TEM, Jeol, Japan) at $200 \mathrm{kV}$. For TEM measurement, one drop of the ethanol dispersion of the MSN powders was placed on a carbon-coated copper grid and then dried at room temperature in air. SAXS measurements were carried out with a Nanostar U small-angle X-ray scattering system (Bruker, Germany) using $\mathrm{Cu} \mathrm{K} \alpha$ radiation $(40 \mathrm{kV}, 35 \mathrm{~mA})$. The porosity of MSN was assessed by a nitrogen adsorptiondesorption measurement. The measurement was operated at 77 K using a Tristar 3020 analyzer (Micromeritics, USA). The pore size distribution was calculated according to the desorption branch of the isotherm using the BJH formula. The BrunauerEmmett-Teller (BET) specific surface area $\left(S_{\mathrm{BET}}\right)$ was obtained on the basis of the nitrogen adsorption data in the relative pressure $\left(P / P_{0}\right)$ ranging from 0.04 to 0.1 .

\subsection{Drug loading and in vitro drug release}

For the drug loading and release, MSN with different pore sizes but similar particle sizes and surface charges were chosen as the nanocarriers. Each kind of MSN ( $3 \mathrm{mg}$ ) was dispersed in $1 \mathrm{~mL}$ of DOX solution $\left(1 \mathrm{mg} \mathrm{mL} \mathrm{mL}^{-1}\right.$ ) in $0.2 \mathrm{M}$ phosphate buffer saline (PBS, pH 7.4). The mixture was sonicated at $200 \mathrm{~W}$ for $30 \mathrm{~min}$ in ice bath, collected by centrifugation, and washed for several times with water. The amount of free DOX in the supernatant was determined by fluorimetry $\left(\lambda_{\mathrm{ex}}=488 \mathrm{~nm}, \lambda_{\mathrm{em}}=598 \mathrm{~nm}\right)$. The encapsulation efficiency (EE) and loading capability (LC) of DOX was calculated from the following equation:

$$
\begin{gathered}
\operatorname{EE~}(\%)=\left(W_{0}-W_{1}\right) / W_{0} \times 100 \\
\operatorname{LC}(\%)=\left(W_{0}-W_{1}\right) /\left(W_{0}-W_{1}+3000 \mu \mathrm{g}\right) \times 100
\end{gathered}
$$

where $W_{1}$ and $W_{0}$ referred to the amount of free DOX $(\mu \mathrm{g})$ and total DOX $(\mu \mathrm{g})$, respectively.

For in vitro drug release experiment, $1 \mathrm{~mL}$ of DOX/MSN suspension ( $1 \mathrm{mg} \mathrm{mL}^{-1}$ ) was put into a dialysis bag (MWCO $3.5 \mathrm{kD}$ ), which was soaked in $50 \mathrm{~mL}$ of $0.2 \mathrm{M}$ PBS (pH 7.4) and incubated at $37{ }^{\circ} \mathrm{C}$ and $100 \mathrm{rpm}$. At predetermined time intervals, $1 \mathrm{~mL}$ of aliquot was taken out to determine the amount of released DOX with fluorimetry $\left(\lambda_{\mathrm{ex}}=488 \mathrm{~nm}, \lambda_{\mathrm{em}}=598 \mathrm{~nm}\right)$, instead of adding an equal volume of fresh release medium.

\subsection{Cellular uptake}

QGY-7703 cells were seeded in 24-well plates at $1 \times 10^{5}$ cells per well at $37{ }^{\circ} \mathrm{C}$ in $5 \% \mathrm{CO}_{2}$ for $24 \mathrm{~h}$. DOX/MSN was added to each well at the concentration of ( $5 \mu \mathrm{g} \mathrm{mL}{ }^{-1}$ DOX eq.) and incubated for $4 \mathrm{~h}$. The cells were washed with PBS and lysed with $0.5 \%(\mathrm{w} /$ v) sodium dodecyl sulfate (SDS, $\mathrm{pH}$ 8.0). Subsequently, the cell lysate was quantified for DOX with fluorimetry $\left(\lambda_{\mathrm{ex}}=488 \mathrm{~nm}\right.$, 
$\lambda_{\mathrm{em}}=598 \mathrm{~nm}$ ) and protein content with the Lowry method. Results were presented as the amount of DOX of $1 \mathrm{mg}$ cellular protein.

\subsection{Nucleic accumulation}

For quantitative analysis, fractional centrifugation was used to separate the nuclei and cytoplasma. QGY-7703 cells were seeded in 6-well plates at $2 \times 10^{5}$ cells per well for $24 \mathrm{~h}$ and incubated with DOX/MSN (5 $\mu \mathrm{g} \mathrm{mL}{ }^{-1}$ DOX eq.) for $4 \mathrm{~h}$. The cells were washed with PBS, lysed with $0.5 \%$ (w/v) SDS (pH 8.0), and centrifugated at $800 \mathrm{rpm}$ for $10 \mathrm{~min}$. The supernatant was collected to calculate the amount of DOX in the cytoplasma. The pellet was resuspended in the lysis buffer to quantify the nucleic contents. The level of DOX in the nuclei and cytoplasma was presented as the percentage associated with the total amount of internalized DOX.

For qualitative analysis, QGY-7703 cells were seeded on coverslips in 6-well plates at $1 \times 10^{5}$ cells per well for $24 \mathrm{~h}$. Following treatment with DOX/MSN (5 $\mu \mathrm{g} \mathrm{mL} \mathrm{mL}^{-1}$ DOX eq.) for $4 \mathrm{~h}$, cells were washed with PBS, fixed with $4 \%(\mathrm{w} / \mathrm{v})$ paraformaldehyde, and stained with Hoechst 33 258. The coverslips were observed by a confocal laser scanning microscopy (CLSM, Zeiss, Germany).

\subsection{Cell viability and anti-proliferation}

QGY-7703 cells were seeded in 96-well plates at $1 \times 10^{4}$ cells per well and cultured at $37{ }^{\circ} \mathrm{C}$ in $5 \% \mathrm{CO}_{2}$ for $24 \mathrm{~h}$. The blank MSN were added to each well at a final concentration of 25-100 $\mu \mathrm{g}$ $\mathrm{mL}^{-1}$ and incubated for another $24 \mathrm{~h}$ before MTT assay to determine cell viability. Untreated cells served as $100 \%$ cell viability.

For in vitro anti-proliferation of tumor cell, QGY-7703 cells were seeded in 96-well plates at $1 \times 10^{4}$ cells per well and cultured at $37{ }^{\circ} \mathrm{C}$ in $5 \% \mathrm{CO}_{2}$ for $24 \mathrm{~h}$. Free DOX and DOX/MSN were added to each well at various final concentrations and incubated for $24 \mathrm{~h}$ before MTT assay to calculate the $\mathrm{IC}_{50}$ values.

\subsection{Cell apoptosis}

QGY-7703 cells were seeded in 6-well plates at $1 \times 10^{5}$ cells per well at $37{ }^{\circ} \mathrm{C}$ in $5 \% \mathrm{CO}_{2}$ for $24 \mathrm{~h}$. After incubating with free DOX and DOX/MSN (1 $\mu \mathrm{g} \mathrm{mL}{ }^{-1}$ DOX eq.) for $24 \mathrm{~h}$, the cells were collected, washed with cold PBS, and then double stained with Annexin V-FITC and PI according to the protocol. Live/ apoptotic/necrotic cells were separated through gating the signals collected on an FACS Caliber flow cytometry (Becton Dickinson, USA) using CellQuest software.

\subsection{In vivo anticancer efficacy}

Mice bearing H-22 tumor were randomly grouped $(n=6)$ when the tumors grew up to $100 \mathrm{~mm}^{3}$. The mice were treated with saline (control), blank MSN at $20 \mathrm{mg} \mathrm{kg}^{-1}$, free DOX at $4 \mathrm{mg}$ $\mathrm{kg}^{-1}$, and DOX/MSN (4 mg kg ${ }^{-1}$ DOX eq.). Drug administration was carried out three times via tail vein injection at day 0 , day 2 , and day 4 . The long diameter $(\mathrm{L}, \mathrm{mm})$ and the short diameter $(\mathrm{S}$, $\mathrm{mm}$ ) of the tumor as well as the body weight of mice were measured every other day. The tumor volume was calculated according to the formula $\left(L \times S^{2}\right) / 2$. At day 16 , the mice were sacrificed and the tumors were excised and weighed. Tumor inhibition ratio (TIR) was calculated as follows:

$$
\operatorname{TIR}=\left(W_{\mathrm{C}}-W_{\mathrm{T}}\right) / W_{\mathrm{C}}
$$

where $W_{\mathrm{T}}$ and $W_{\mathrm{C}}$ presented the average tumor weight of the treatment and control group, respectively.

\subsection{Statistical analysis}

Statistical analysis was conducted using student's $t$ test between two groups or single factor analysis of variance (ANOVA) among three or more groups. The differences were judged to be significant at $P<0.05$.

\section{Results and discussion}

\subsection{Preparation and characterization of MSN}

MSN were synthesized by a microemulsion method. This stratification approach allowed the reaction to conduct in the interface since it was easy to control the combination on the interface by means of adding or changing reactants without destroying the traction interface. In a one-pot interfacial growth process, the pore size could be tuned by changing hydrophobic solvents. The MSN with different pore sizes were characterized by SAXS, nitrogen adsorption-desorption isotherms, DLS, and TEM. In SAXS patterns, a single scattering peak could be noted, indicating that the three kinds of MSN had uniform mesostructure $^{\mathbf{1 3 , 1 5}}$ (Fig. 1A). Additionally, the scattering peaks of all MSN at different $q$ values implied their differences in pore sizes. ${ }^{16}$ Three MSN exhibited type IV nitrogen adsorptiondesorption isotherms according to IUPAC nomenclature (Fig. 1B). Such type IV isotherm was representative of a cylindrical and channel-like mesoporous structure. At around $0.5<$ $P / P_{0}<0.75$, a capillary condensation step revealed narrow pore size distribution of all MSN. Similar isotherm curves implied that various MSN possessed identical pore structures, which ensured the follow-up single variable analysis of pore size. ${ }^{17}$ The pore size distribution curve with single peak demonstrated each MSN had only one modal pore (Fig. 1C). Table 1 summarized particle sizes, surface charges, and the BET-quantified pore structures of MSN. DLS measurement showed that the MSN possessed particle sizes of about $130 \mathrm{~nm}$. The phosphonatecoated MSN exhibited a highly negative surface charge of around $-35 \mathrm{mV}$, which allowed an electrostatic attachment to positively charged DOX. The BET-quantified pore structures of the MSN included pore size, pore volume, and surface area. The pore size highly depended on the type of the hydrophobic solvent in upper oil and the concentration of TEOS. When the concentration of TEOS in 1-octadecene was $20 \%(\mathrm{v} / \mathrm{v})$, the MSN with small pore size of $2.3 \mathrm{~nm}$ (MSN2) could be achieved. However, when the solvent was replaced with cyclohexane, the MSN with medium pore size of $5.4 \mathrm{~nm}$ (MSN5) could be realized. After diluting the concentration of TEOS to $5 \%(\mathrm{v} / \mathrm{v})$ in cyclohexane, the MSN with large pore size of $8.2 \mathrm{~nm}$ (MSN8) were obtained. The volume of pores and BET surface area of 
A

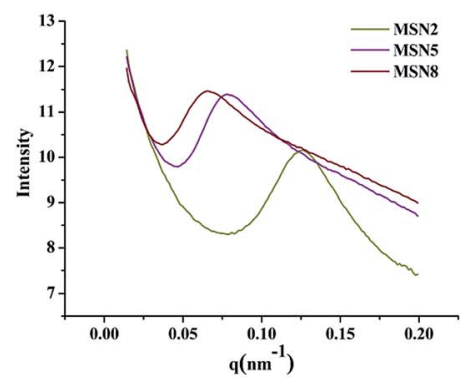

B

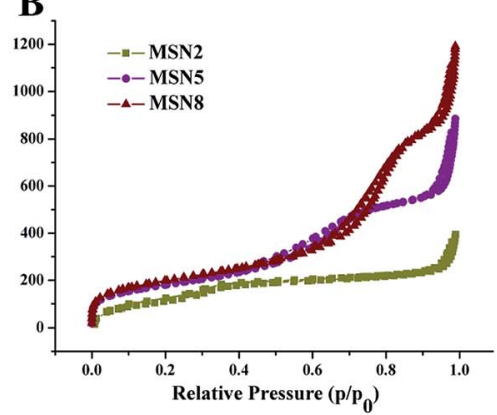

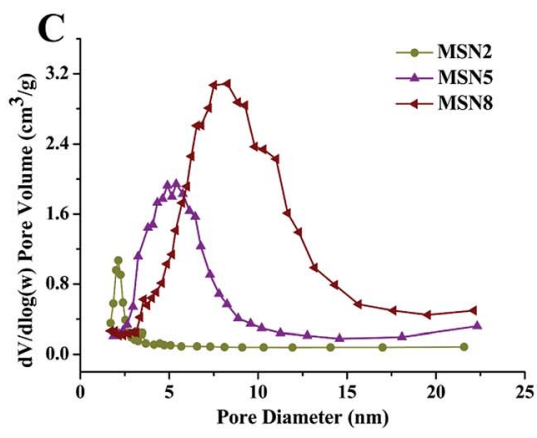

Fig. 1 SAXS patterns (A), nitrogen adsorption-desorption isotherms (B), and corresponding pore size distributions (C) of MSN.

Table 1 Particle sizes, surface charges, and the BET-quantified pore structures of various MSN

\begin{tabular}{|c|c|c|c|c|c|}
\hline Sample & Particle size $^{a}(\mathrm{~nm})$ & Surface charge $^{a}(\mathrm{mV})$ & BET surface $\operatorname{area}^{b}\left(\mathrm{~m}^{2} \mathrm{~g}^{-1}\right)$ & Volume of pores ${ }^{c}\left(\mathrm{~cm}^{3} \mathrm{~g}^{-1}\right)$ & Mean size of pores ${ }^{c}(\mathrm{~nm})$ \\
\hline MSN2 & $133.5 \pm 6.8,(0.159)$ & $-38.2 \pm 4.2$ & 423 & 0.492 & 2.3 \\
\hline MSN5 & $133.9 \pm 13.0,(0.057)$ & $-35.4 \pm 3.2$ & 650 & 1.229 & 5.4 \\
\hline
\end{tabular}

${ }^{a}$ Particle sizes and surface charges were determined by DLS measurement. Indicated values were mean \pm SD $(n=3)$. Values in parentheses represent the polydispersity index (PDI). ${ }^{b}$ Obtained from BET method. ${ }^{c}$ Obtained from BJH method.

MSN increased as the pore size increased. Such results might be ascribed to various swelling behaviors of oil-water stratification reaction system in the preparation procedure of MSN arising from different molecular sizes and hydrophobicities of organic solvent.

The morphology and porous structure of MSN were observed by TEM. As shown in Fig. 2, all MSN were highly uniform nanospheres and had homogeneous center-radial pore channels. TEM observation showed an increasing pore size in the order of MSN2 < MSN5 < MSN8, which was in agreement with the BET-quantified pore structures.

\subsection{Drug loading and in vitro drug release}

The mesoporous was one of the advantages of MSN for drug delivery systems, which could easily and effectively load a wide range of therapeutic drugs. As listed in Table 2, among three kinds of MSN, MSN2 had the lowest EE and LC of DOX, which might be due to their small pore size, pore volume, and surface area. ${ }^{18}$ MSN5 exhibited the similar EE and LC of DOX with MSN8. Such results were attributed to enough pore size of
MSN5, which led to easier binding and diffusion of DOX inside the pores. Although MSN8 had larger pore size, already-loaded DOX might restrict a further absorption of pore occupation/ filling, thus resulting in an underutilization of the total pore volume. ${ }^{19}$ In general, MSN with enough pore size exhibited more loaded drugs into the pores owing to better packing efficiency of the drug inside the wider pores. Our preliminary experiments indicated that the surface charge could affect the loading of DOX. Since DOX was positively charged at the loading condition utilized, lower amount of DOX was loaded into MSN bearing positive charge compared to the negatively charged ones, irrespective of their similar pore sizes and pore structures. This implied that the ionic interaction between DOX and MSN also played an important role in determining the LC. Therefore, in this study, DOX and MSN dissolved in PBS ( $\mathrm{pH}=7.4)$ bore positive and negative charges, respectively, which was favorable for the enhancement of LC of DOX.

Fig. 3 depicted the drug release profiles of DOX/MSN. It could be seen that all DOX/MSN had an initial burst, followed by a prolonged release up to $24 \mathrm{~h}$, which would be beneficial to the
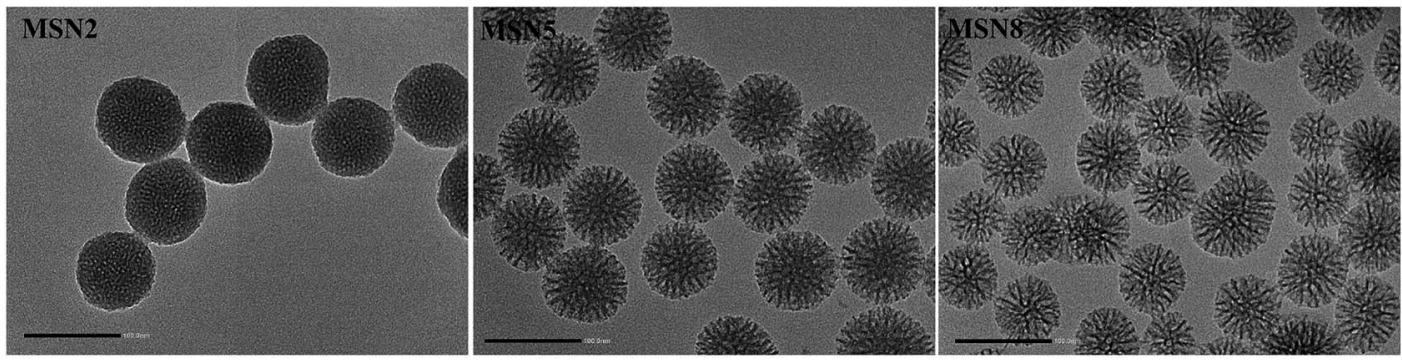

Fig. 2 TEM images of MSN (scale bar $=100 \mathrm{~nm}$ ). 
Table 2 EE and LC of various DOX/MSN

\begin{tabular}{lcc}
\hline Sample & EE $(\%)$ & LC $(\%)$ \\
\hline DOX/MSN2 & $74.5 \pm 0.1$ & $8.2 \pm 0.2$ \\
DOX/MSN5 & $96.0 \pm 0.1^{a}$ & $21.1 \pm 0.1^{a}$ \\
DOX/MSN8 & $96.5 \pm 0.1^{a}$ & $21.1 \pm 0.1^{a}$ \\
${ }^{a} P<0.01$, compared to DOX/MSN2. &
\end{tabular}

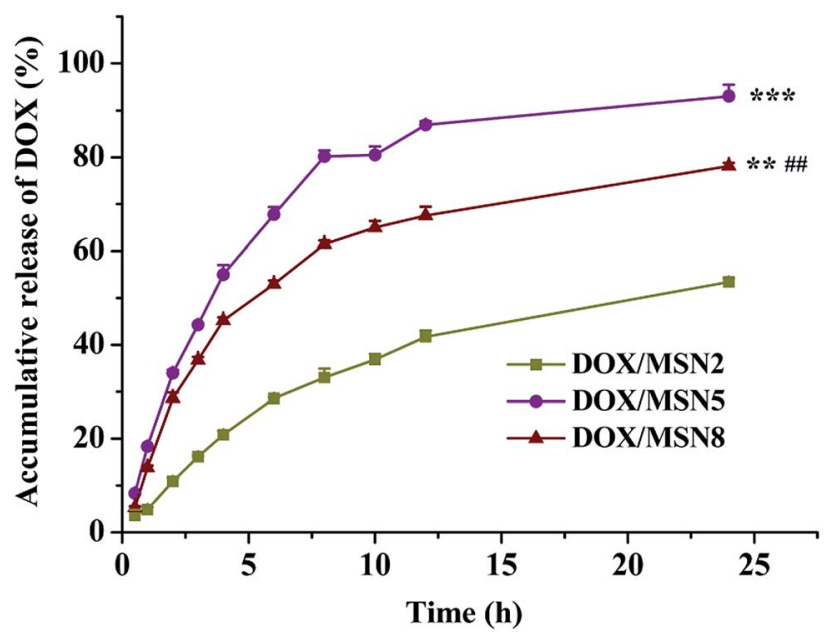

Fig. 3 In vitro drug release of DOX/MSN in $0.2 \mathrm{~mol} \mathrm{~L}^{-1} \mathrm{PBS}$. Indicated values were mean $\pm \mathrm{SD}(n=3) . * * P<0.01, * * * P<0.001$ vs. DOX/ MSN2, ${ }^{\# \#} P<0.01$ vs. DOX/MSN5.

need for immediate treatment after administration and the maintenance of effective drug concentration in the blood, respectively. The release rates and $24 \mathrm{~h}$ accumulative release percentages of all DOX/MSN were in the order of DOX/MSN2 < DOX/MSN8 < DOX/MSN5. The slower release rate of DOX/MSN2 was attributed to the slower diffusion of the solvent into the smaller pores and slower counter diffusion of the drug to the bulk solution. Since the pore size of MSN5 was smaller than that of MSN8, DOX loaded into them experienced more restricted diffusion, which resulted in shorter diffusion distance arising from a larger number of DOX locating close to the pore entrances, thereby showing a more rapid release rate than DOX/ MSN8. MSN8 with large pore size might load DOX into deeper pore channel, thus leading to longer diffusion distance and slower release rate..$^{20}$ Although a large pore size could allow for easy accommodation of the guest molecular, it did not guarantee efficient release kinetics. ${ }^{21,22}$ Therefore, MSN5 with the medium pore size would be an appropriate drug carrier in view of higher LC and more efficient in vitro release behavior.

\subsection{Cellular uptake}

High level of cellular uptake is the prerequisite for intracellular drug delivery. The cellular internalization of DOX/MSN was measured in liver cancer QGY-7703 cells by fluorimetry. As indicated in Fig. 4, the maximum in vitro uptake amount was detected in the DOX/MSN5-treated group, which was 3.2- and

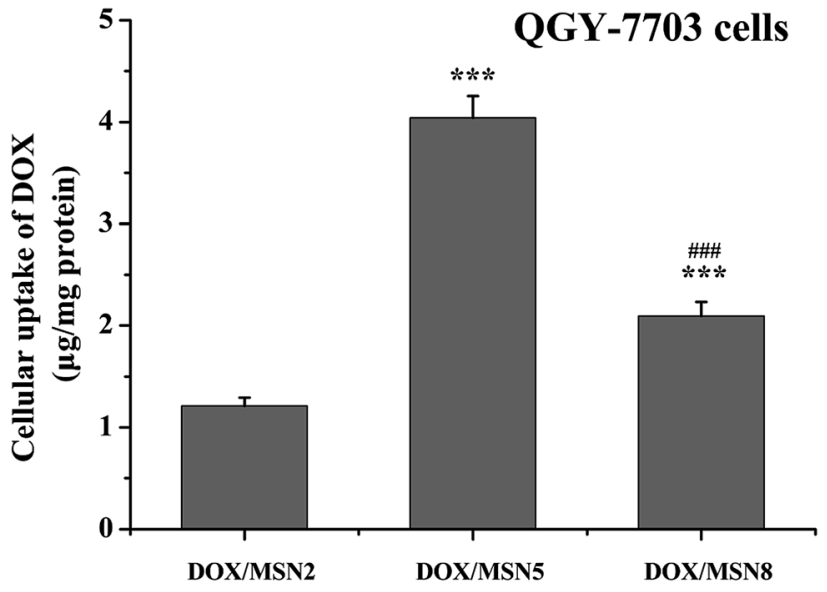

Fig. 4 Cellular uptake of DOX/MSN in QGY-7703 cells following incubation for $4 \mathrm{~h}$. Indicated values were mean $\pm \mathrm{SD}(n=3)$. ${ }^{* * *} P<$ 0.001 vs. DOX/MSN2. ${ }^{\# \#} P<0.001$ vs. DOX/MSN5.

1.9-folds higher than that of DOX/MSN2 and DOX/MSN8, respectively. Once arriving into tumor cells, DOX loaded in MSN tended to be delivered into the cells in the encapsulated and released form. The released DOX can readily enter the tumor cells via diffusion. Therefore, the rapid drug release profile of DOX/MSN5 could be responsible for their higher cellular uptake.

\subsection{Nucleic accumulation}

As DOX was required entering into the nuclei to conduct anticancer activity, the nucleic accumulation of DOX/MSN would highly influence their in vitro and in vivo anticancer activity. ${ }^{23}$ The cytoplasmic and nucleic distribution of DOX/MSN were quantitatively and qualitatively investigated by fractional centrifugation and observation with CLSM, respectively (Fig. 5A and B). As depicted in Fig. 5A, the relative nucleic accumulation fractions of DOX/MSN were in the order of DOX/MSN2 < DOX/ MSN8 < DOX/MSN5, indicating the pore size of MSN played an important role in the nucleic transportation of DOX. Such results might be due to the higher cellular uptake level and more efficient drug release profile of DOX/MSN5. Qualitative CLSM observations further confirmed the results of quantitative analysis (Fig. 5B), which also suggested the higher cellular uptake and nucleic accumulation of DOX/MSN5.

\subsection{Cell viability and anti-proliferation}

Prior to investigating their potential applications as drug delivery carriers, the cytotoxicity of blank MSN had to be evaluated. As shown in Fig. 6A, blank MSN exhibited negligible cell mortality on QGY-7703 cells at the concentration up to $100 \mu \mathrm{g}$ $\mathrm{mL}^{-1}$, excluding their non-specific cytotoxicity. A possible explanation for this behavior could be the fact that negatively charged surface of MSN minimize the interaction between MSN and negatively charged cell membrane, thus avoiding the impairment of critical membrane functions and intracellular process. $^{24}$ 
A

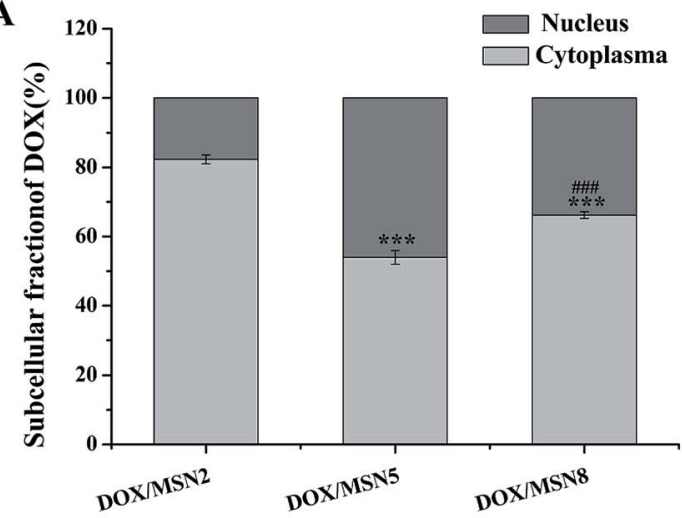

B

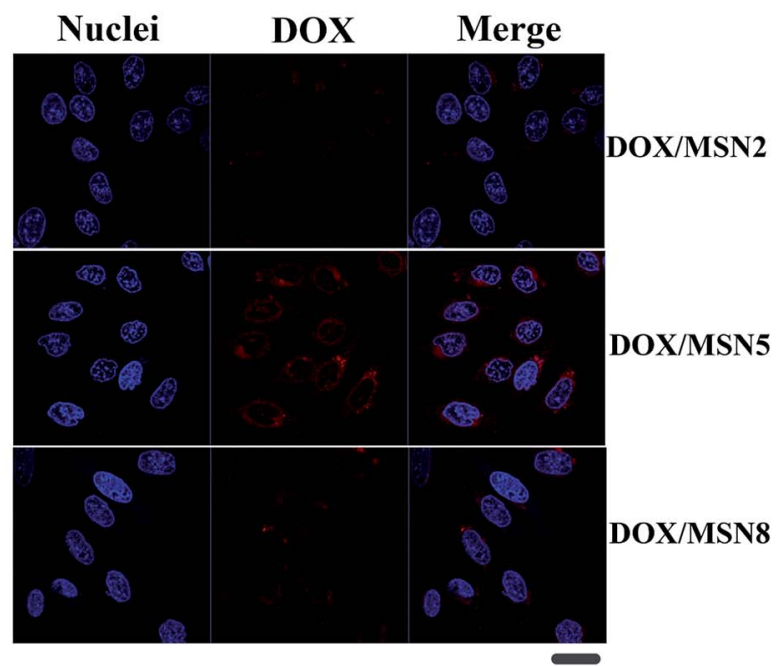

Fig. 5 Intracellular distribution of DOX in QGY-7703 cells following treatment with DOX/MSN at $37^{\circ} \mathrm{C}$ for $4 \mathrm{~h}$. Indicated values were mean

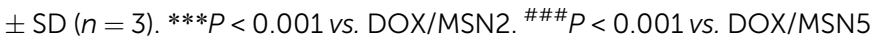
(A) and CLSM images of QGY-7703 cells incubated with DOX/MSN for $4 \mathrm{~h}$. Bar represented $20 \mu \mathrm{m}$ (B).
To demonstrate the effects of DOX/MSN-induced anticancer therapy, an in vitro anti-proliferation assay was carried out. The half-maximal inhibitory concentration $\left(\mathrm{IC}_{50}\right)$ values of free DOX, DOX/MSN2, DOX/MSN5, and DOX/MSN8 against QGY-7703 cells following $24 \mathrm{~h}$ treatment were calculated to be $2.70,1.56$, 0.60 , and $1.16 \mu \mathrm{g} \mathrm{mL} \mathrm{m}^{-1}$, respectively (Fig. 6B). Compared with free DOX, DOX/MSN showed remarkable cytotoxicity, indicating that DOX loaded into MSN might be more efficiently delivered into nuclei than free DOX. The inhibition of cell growth was closely related to the pore sizes of MSN, wherein DOX/MSN5 exhibited the strongest in vitro anti-proliferation of tumor cells among all the DOX/MSN, which might be ascribed to their rapid and complete release behavior, high cellular uptake, and preferable nucleic accumulation of DOX.

\subsection{Cell apoptosis}

The AnnexinV-FITC/PI double staining assay was carried out to make a distinction between apoptosis cells and necrosis cells induced by free DOX and DOX/MSN. As illustrated in Fig. 7, the lower left, lower right, and upper right quadrants in the quadrantal diagram represented live, early apoptotic, and late apoptotic/necrotic cells, respectively. After $24 \mathrm{~h}$ treatment, a negligible percentage of apoptosis and necrosis was detected for cells without treatment. In contrast, the percentage of apoptotic QGY-7703 cells induced by DOX/MSN2, DOX/MSN5, DOX/MSN8, and free DOX were 32.09\%, 72.55\%, 43.40\%, and $13.89 \%$, respectively, when the concentration of DOX was $1 \mu \mathrm{g}$ $\mathrm{mL}^{-1}$, which was in agreement with the in vitro antiproliferation activity.

\subsection{In vivo anticancer efficacy}

To our best knowledge, few studies of MSN focused on how the pore size of MSN impacted on in vivo anticancer efficacy. To this end, DOX/MSN with various pore sizes were intravenously injected into tumor-bearing mice. As depicted in Fig. 8A, gradually increased body weight was noted for all treated groups, indicating desirable safety profile of MSN. As shown in Fig. 8B
A

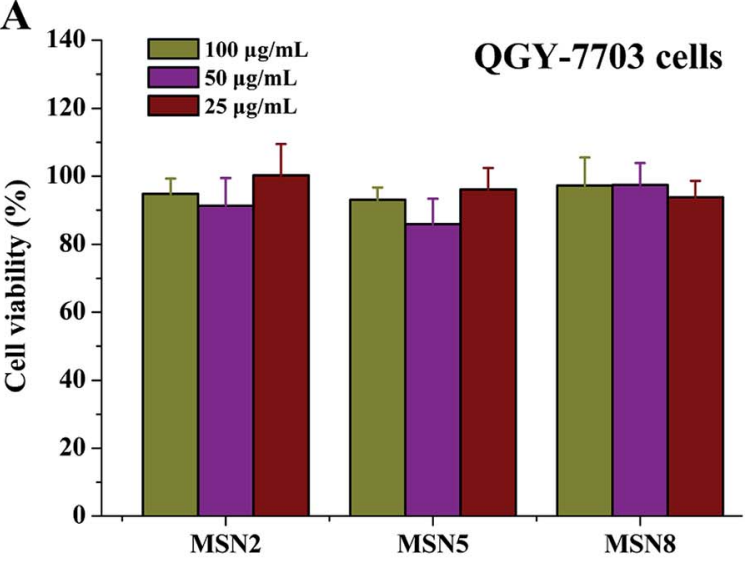

B

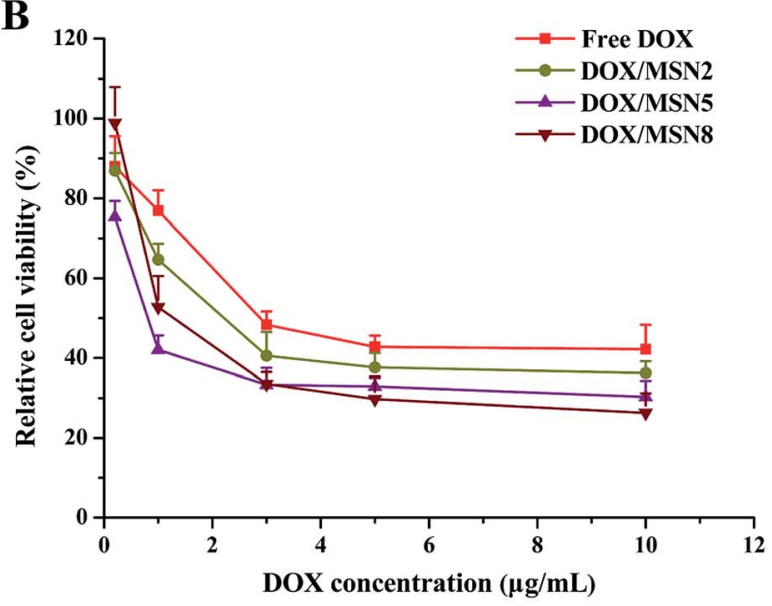

Fig. 6 Viability of QGY-7703 cells after incubation with blank MSN (A) and anti-proliferation of QGY-7703 cells after incubation with free DOX and DOX/MSN (B) at various concentrations for $24 \mathrm{~h}$. Indicated values were mean \pm SD $(n=6)$. 

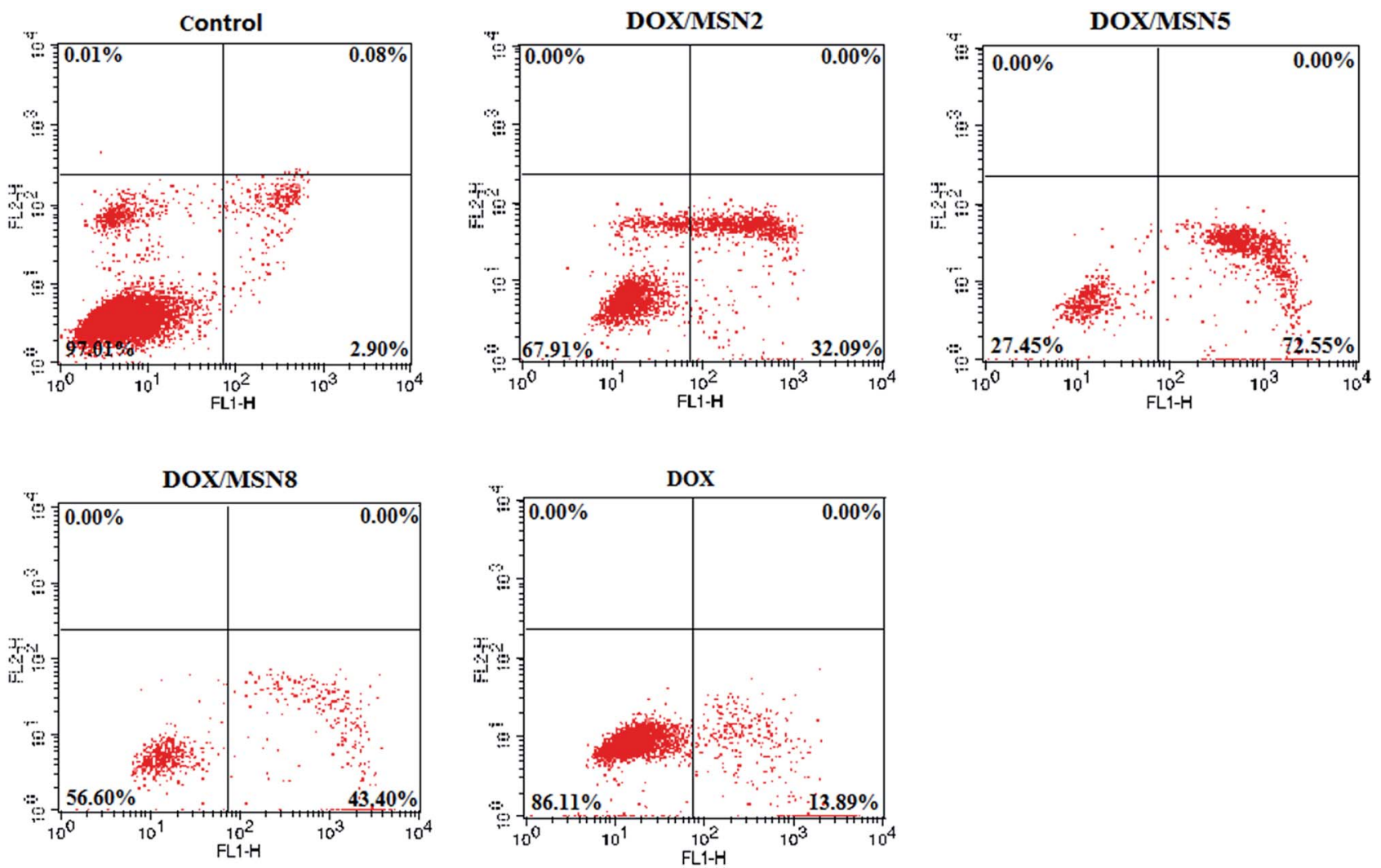

Fig. 7 Evaluation on the apoptosis of QGY-7703 cells treated with free DOX and DOX/MSN by flow cytometry.
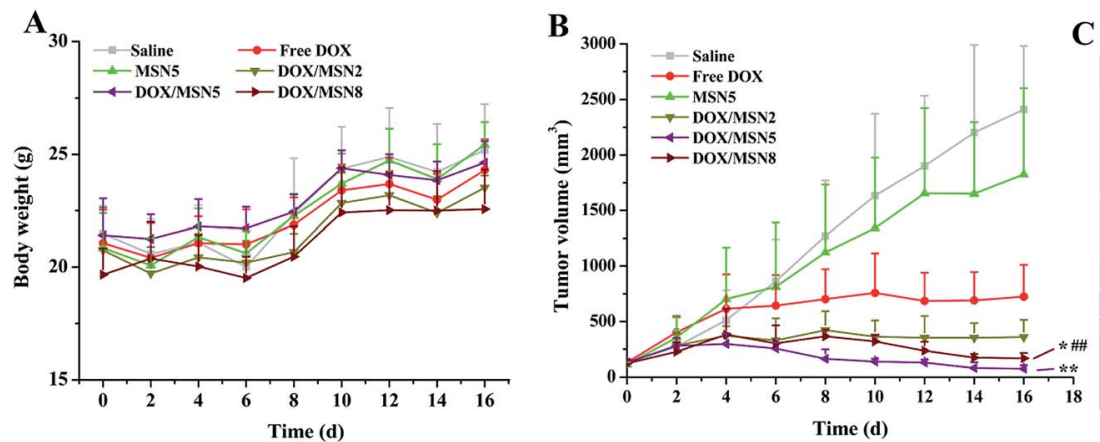

C

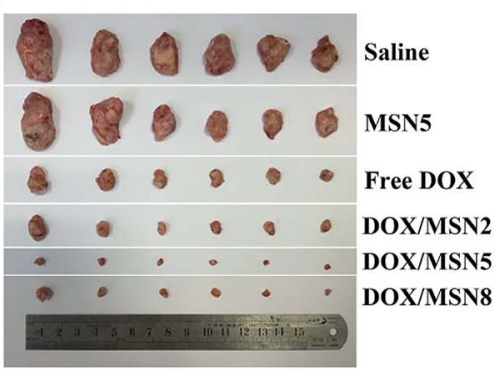

Fig. 8 The in vivo anticancer efficacies of MSN. (A) The body weight growth curves and (B) tumor growth curves of $\mathrm{H}-22$ tumor-bearing mice in various therapeutic groups during the 16 days' experimental period. Indicated values were mean \pm SD $(n=6) . * P<0.05, * * P<0.01$ vs. DOX/ MSN2. ${ }^{\# \#} P<0.01$ vs. DOX/MSN5. (C) The photograph of excised tumors at day 16.

and C, although the blank MSN exhibited some tumor growth inhibition compared to the control saline, there is no statistically significant differences $(P>0.05)$, suggesting the inefficacy of bank MSN. On the contrary, DOX/MSN and free DOX exerted significantly impeded tumor growth to different degrees. Better anticancer capabilities of DOX/MSN (TIR of 91.6-98.9\%) were observed as compared with free DOX (TIR of $84.0 \%$ ), which might be attributed to their increased tumor accumulations through the enhanced permeability and retention (EPR) effect. As compared with DOX/MSN2 and DOX/MSN8, DOX/MSN5 exhibited the stronger tumor inhibition, in accordance with the results of in vitro cellular uptake, anti-proliferation assay, nucleic accumulation, and cell apoptosis assay. This phenomenon might result from their suitable pore sizes for drug loading and release. These results collectively apprehended that pore size of MSN played an important role in the delivery systems of anticancer drugs, wherein MSN with suitable pore sizes preferred achieving an outstanding performance in the in vitro and in vivo therapeutic efficacy.

\section{Conclusions}

In this study, three resultant MSN with different pore sizes were successfully synthesized. MSN5 with medium pore size 
possessed a higher LC of DOX as well as more rapid and complete drug release profiles. Compared with DOX/MSN2 and DOX/MSN8, DOX/MSN5 exhibited higher cellular uptake and nucleic accumulation, thereby accounting for their stronger in vitro anti-proliferation and apoptosis of tumor cells and in vivo tumor growth retardation. These results suggested that efforts to design a reasonable pore size of MSN would be an important subject to achieve optimal anticancer efficacies.

\section{Conflicts of interest}

There are no conflicts to declare.

\section{Acknowledgements}

The authors are thankful for the grants from National Natural Science Foundation of China (No. 81273460).

\section{References}

1 A. H. Faraji and P. Wipf, Bioorg. Med. Chem., 2009, 17, 19502962.

2 M. E. Davis, Z. Chen and D. M. Shin, Nat. Rev. Drug Discovery, 2008, 7, 771-782.

3 P. A. Ma, H. H. Xiao, C. X. Li, Y. L. Dai, Z. Y. Cheng, Z. Y. Hou and J. Lin, Mater. Today, 2015, 18, 554-564.

4 J. L. Vivero-Escoto, I. I. Slowing, B. G. Trewyn and V. S. Y. Lin, Small, 2010, 6, 1952-1967.

5 Y. Wang, Q. F. Zhao, N. Han, L. Bai, J. Li, J. Liu, E. X. Che, L. Hu, Q. Zhang and T. Y. Jiang, Nanomedicine, 2015, 11, 313-327.

6 D. P. Otto, A. Otto and M. M. de Villiers, Expert Opin. Drug Delivery, 2015, 12, 763-777.

7 A. Yadollahpour and S. Rashidi, Orient. J. Chem., 2015, 31, 25-30.

8 N. Ž. Kneževic and J. O. Durand, Nanoscale, 2015, 7, 21992209.

9 M. Fujiwara, F. Yamamoto, K. Okamoto, K. Shiokawa and R. Nomura, Anal. Chem., 2005, 77, 8138-8145.
10 Y. Gao, Y. Chen, X. F. Ji, X. Y. He, Q. Yin, Z. W. Zhang, J. L. Shi and Y. P. Li, ACS Nano, 2011, 5, 9788-9798.

11 A. Takimoto, T. Shiomi, K. Ino, T. Tsunoda, A. Kawai, F. Mizukami and K. Sakaguchi, Microporous Mesoporous Mater., 2008, 116, 601-606.

12 L. J. Jia, J. Y. Shen, Z. Y. Li, D. R. Zhang, Q. Zhang, C. X. Duan, G. P. Liu, D. D. Zheng, Y. Liu and X. N. Tian, Int. J. Pharm., 2012, 439, 81-91.

13 D. K. Shen, J. P. Yang, X. M. Li, L. Zhou, R. Y. Zhang, W. Li, L. Chen, R. Wang, F. Zhang and D. Y. Zhao, Nano Lett., 2014, 14, 923-932.

14 H. Meng, W. X. Mai, H. Y. Zhang, M. Xue, T. Xia, S. J. Lin, X. Wang, Y. Zhao, Z. X. Ji, J. I. Zink and A. E. Nel, ACS Nano, 2013, 7, 994-1005.

15 Z. X. Li, L. L. Zhang, C. Tang and C. H. Yin, Pharm. Res., 2017, 34, 2829-2841.

16 L. Robben, A. A. Ismail, D. W. Bahnemann and J. C. Buhl, Microporous Mesoporous Mater., 2011, 143, 277-283.

17 L. Rashidi, E. Vasheghani-Farahani, K. Rostami, F. Ganji and M. Fallahpour, Asia-Pac. J. Chem. Eng., 2014, 9, 845-853.

18 J. X. Zhang, X. Li, J. M. Rosenholm and H. C. Gu, J. Colloid Interface Sci., 2011, 361, 16-24.

19 J. Zhang, M. Niemela, J. Westermarck and J. M. Rosenholm, Dalton Trans., 2014, 43, 4115-4126.

20 J. O. Martinez, C. Chiappini, A. Ziemys, A. M. Faust, M. Kojic, X. W. Liu, M. Ferrari and E. Tasciotti, Biomaterials, 2013, 34, 8469-8477.

21 N. H. N. Kamarudin, A. A. Jalil, S. Triwahyono, N. F. M. Salleh, A. H. Karim, R. R. Mukti, B. H. Hameed and A. Ahmad, Microporous Mesoporous Mater., 2013, 180, 235-241.

22 Q. L. Li, Y. F. Sun, Y. L. Sun, J. J. Wen, Y. Zhou, Q. M. Bing, L. D. Isaacs, Y. H. Jin, H. Gao and Y. W. Yang, Chem. Mater., 2014, 26, 6418-6431.

23 F. Yang, S. S. Teves, C. J. Kemp and S. Henikoff, Biochim. Biophys. Acta, Rev. Cancer, 2014, 1845, 84-89.

24 Y. N. Zhao, X. X. Sun, G. N. Zhang, B. G. Trewyn, I. I. Slowing and V. S. Y. Lin, ACS Nano, 2011, 5, 1366-1375. 